\title{
Study of mass transfer correlations for rotating packed bed columns in the context of solvent-based carbon capture
}

\author{
Eni Oko, Meihong Wang*, Colin Ramshaw \\ Department of Chemical and Biological Engineering, University of Sheffield, S1 3JD, UK \\ *Corresponding Author: Tel.: +44 1114222 7160. E-mail address: Meihong.Wang@ @heffield.ac.uk
}

\begin{abstract}
The application of rotating packed beds (RPBs) in solvent-based carbon capture processes, will greatly reduce the physical footprint, capital and operating cost of the process. However, in designing RPBs, correlations for predicting mass transfer parameters are generally limited in literature and their prediction accuracies have not been demonstrated independently. In this paper, an RPB absorber model was developed in gPROMS ModelBuilder $^{\circledR}$ and used to test and compare different correlations for predicting the effective interfacial area, liquid and gas film mass transfer coefficients. Our results showed that the modified packed column mass transfer correlations where the " $g$ " term (i.e. gravitational acceleration) is replaced with " $r w^{2}$ " (i.e. centrifugal acceleration) commonly used in literature for RPBs generally give poor predictions compared to using correlations developed specifically for RPBs. Also, the Tung and Mah correlation has better predictive accuracy for the liquid film mass transfer coefficient in RPBs than more complex correlations. Finally, a set of new data for the gas film mass transfer coefficient for RPBs were also derived from overall volumetric mass transfer coefficient $\left(K_{G} a\right)$ experimental data from literature. This is the first report of gas film mass transfer data for RPBs. The results in this paper will guide researchers in selecting suitable correlations for predicting mass transfer parameters in RPBs.
\end{abstract}

Keywords: solvent-based $\mathrm{CO}_{2}$ capture; rotating packed bed; effective interfacial area; liquid film mass transfer coefficient; gas film mass transfer coefficient

\section{Nomenclature}

$a$

$a_{t}$

$A$

$c, d$

$C_{p}^{L}$

$d_{h}$

$d_{p}$

$D_{L}$

$D_{G}$

E

$G^{m}$

$h_{G}$

$h_{L}$

$h_{g / l}$

H

$\Delta H_{r}$

$\Delta H_{\text {vap }}$

$k_{G}$

$K_{G} a$

$k_{L}$

$K_{L} a$

$k_{\text {app }}$

$L^{m}$

$L_{m}^{*}$

$N_{i}$

$Q_{L}$

$Q_{V}$
Effective interfacial area of packing per unit volume $\left(\mathrm{m}^{2} / \mathrm{m}^{3}\right)$

Total area of packing per unit volume $\left(\mathrm{m}^{2} / \mathrm{m}^{3}\right)$

Tangential section area $\left(\mathrm{m}^{2}\right)=2 \pi r Z$

Packing parameters for Luo et al. (2012a) correlation $(c=3.5 \mathrm{~mm}, d=1.0 \mathrm{~mm})$

Liquid specific heat capacity $(\mathrm{J} / \mathrm{kg} \mathrm{K})$

Hydraulic diameter $(\mathrm{m})=4 \epsilon / a_{t}$

Effective diameter of packing $(\mathrm{m})=6(1-\epsilon) / a_{t}$

Liquid diffusivity $\left(\mathrm{m}^{2} / \mathrm{s}\right)$

Gas diffusivity $\left(\mathrm{m}^{2} / \mathrm{s}\right)$

Enhancement factor

Gas molar flowrate (kmol/s)

Gas phase specific molar enthalpy $(\mathrm{J} / \mathrm{kmol})$

Liquid phase specific molar enthalpy $(\mathrm{J} / \mathrm{kmol})$

Interfacial heat transfer coefficient $\left(\mathrm{W} / \mathrm{m}^{2} \mathrm{~K}\right)$

Henry constant

Heat of absorption $(\mathrm{J} / \mathrm{kmol})$

Heat of vaporisation of $\mathrm{H}_{2} \mathrm{O}(\mathrm{J} / \mathrm{kmol})$

Gas film mass transfer coefficient $(\mathrm{m} / \mathrm{s})$

Overall volumetric mass transfer coefficient based on gas side (1/s)

Liquid film mass transfer coefficient $(\mathrm{m} / \mathrm{s})$

Overall volumetric mass transfer coefficient based on liquid side (1/s)

Apparent reaction rate constant

Liquid molar flowrate $(\mathrm{kmol} / \mathrm{s})$

Liquid mass flowrate per unit tangential section area $\left(\mathrm{kg} / \mathrm{m}^{2} \mathrm{~s}\right)$

Component molar fluxes $\left(\mathrm{kmol} / \mathrm{m}^{2} \mathrm{~s}\right)$

Liquid volumetric flowrate $\left(\mathrm{m}^{3} / \mathrm{s}\right)$

Gas volumetric flowrate $\left(\mathrm{m}^{3} / \mathrm{s}\right)$ 


\section{Greek Letters}

$\sigma_{c}$

$\sigma_{L}$

$\varepsilon$

$\rho_{G}$

$\rho_{L}$

$\lambda_{L}$

$\mu_{G}$

$\mu_{L}$

$\omega$
Radius (m)

Inner radius of the packed bed (m)

Outer radius of the packed bed (m)

Radius of the stationary housing (m)

Revolutions per minute

Gas and liquid side temperature (K)

Liquid velocity $(\mathrm{m} / \mathrm{s})$

Parameter for Chen et al. (2011) gas film model $=1-0.9 \frac{V_{o}}{V_{t}}$

Parameter for Chen et al. (2006) liquid film model $=1-0.93 \frac{V_{o}}{V_{t}}-1.13 \frac{V_{i}}{V_{t}}$

Volume inside the inner radius of the bed $\left(\mathrm{m}^{3}\right)=\pi r_{i}^{2} Z$

Gas mass flowrate per unit tangential section area $\left(\mathrm{kg} / \mathrm{m}^{2} \mathrm{~s}\right)$

Volume between the outer radius of the bed and the stationary housing $\left(\mathrm{m}^{3}\right)=\pi\left(r_{s}^{2}-r_{o}^{2}\right) Z$

Total volume of the RPB $\left(\mathrm{m}^{3}\right)=\pi r_{s}^{2} Z$

Component molar fraction in liquid phase

Component molar fraction in gas phase

Height of the rotor (m)

Critical surface tension for packing material $(=0.075 \mathrm{~N} / \mathrm{m})$

Liquid surface tension $(\mathrm{N} / \mathrm{m})$

Packing porosity $\left(\mathrm{m}^{3} / \mathrm{m}^{3}\right)$

Gas density $\left(\mathrm{kg} / \mathrm{m}^{3}\right)$

Liquid density $\left(\mathrm{kg} / \mathrm{m}^{3}\right)$

Liquid thermal conductivity (W/m K)

Gas dynamic viscosity (Pa s)

Liquid dynamic viscosity (Pa s)

Rotating speed ( $\mathrm{rad} / \mathrm{s})$

\section{Introduction}

\subsection{Background}

The gas-liquid packed columns are an important unit operation in natural gas treating and solvent-based $\mathrm{CO}_{2}$ capture processes where they are used for absorption and desorption. The packed columns in these processes are large in size, contributing significantly to physical footprint, capital and operating costs (Lawal et al., 2012; IEAGHG, 2013; Oko, 2015). An engineering estimate showed that absorbers in a solvent-based $\mathrm{CO}_{2}$ capture (PCC) plant using monoethanolamine (MEA) solvent for capturing $\mathrm{CO}_{2}$ from a $500 \mathrm{MWe}$ coal-fired subcritical power plant will have diameters up to $25 \mathrm{~m}$ and packing height over $27 \mathrm{~m}$ (Oko, 2015). This will significantly increase the land use per MWe when coal and gas fired power plants are integrated with PCC plants (Florin and Fennel, n.d.).

Through process intensification (PI), wherein the packed columns are replaced with rotating packed beds (RPBs), the physical footprint of the process could be reduced significantly (Joel et al., 2014; Thiels et al., 2016). Theoretical investigations by Agarwal et al. (2010) and Joel et al. (2014) showed about 10-12 times reduction in the absorber size when it is replaced with an RPB. The HiGee Environment and Energy Technologies Inc. USA also reported about 10 times size reduction in a commercial scale RPB installed to replace a packed column at the Fujian Refining and Petrochemical Company Ltd, China (HiGee, 2014). The reported size reductions are consistent with predictions about RPBs in earlier investigations by Chambers and Wall (1954) and Ramshaw and Mallinson (1981).

\subsection{Principle of RPB and problem statement}

The RPB generally includes a cased annular packed bed (rotor), made of packing materials such as glass bead (Munjal et al., 1989a\&b), corrugated disk (Chen et al., 1997; Chen et al., 1999), wire mesh (Luo et al., 2012), expamet (Jassim et al., 2007), blade packing with static baffles (Tsai and Chen, 2015), nickel foam (Chu et al., 
2015) etc. and mounted on a rotating shaft (Fig. 1). The gas and liquid phases enter the RPB through the outer and inner sections respectively, each flowing radially as shown in Figure 1 Sectional view of an RPB . 1. The gasliquid flow are usually countercurrent flow, but co-current and cross flow configurations are also possible (Kolawole et al., 2018, Oko et al., 2018). As the RPB rotates, the liquid and gas phases are subjected to intense centrifugal acceleration which is many times the gravitational acceleration in packed columns. As a result, the RPB generally allows:

- Higher flooding limit leading to drastic reduction in packing volume (Guo et al., 1997; Chen et al., 2008; Garcia et al., 2017)

- Lower liquid holdup and consequently achieves steady state more quickly (Nascimento et al., 2009)

- More viscous solvents e.g. 80-100 wt\% MEA solvent (Chambers and Wall, 1954; Jassim et al., 2007; Oko et al. 2018).

Consequently, similar capture levels (in $\mathrm{CO}_{2}$ capture applications) as in packed columns can be achieved in RPBs using significantly reduced packing volume (Agarwal et al., 2010; Joel et al., 2014; Thiels et al., 2016). However, the presence of centrifugal force field in RPBs presents new research challenge as mass transfer correlations for packed columns cannot be used to predict mass transfer in RPBs with acceptable accuracy (Joel et al., 2014; Kang et al., 2014).

Only a few correlations have been reported for predicting effective interfacial area, liquid and gas film mass transfer coefficients (Tung and Mah, 1985; Munjal et al., 1989a; Chen et al., 2006a; Chen et al., 2006b; Chen et al., 2006; Chen, 2011; Rajan et al., 2011; Luo et al., 2012). Modification of mass transfer correlations for packed columns such as Onda et al. (1968) and Billets and Schultes (1999) correlations by replacing the "g" term (i.e.

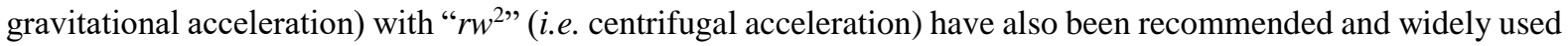
(Joel et al., 2014; Kang et al., 2014; Thiels et al., 2016). There has not been a clear independent demonstration of the performance of the various mass transfer correlations for RPBs against experimental data. This will highlight the strengths and weaknesses of various options and provide a basis for determining the most accurate option for predicting mass transfer parameters in RPBs.

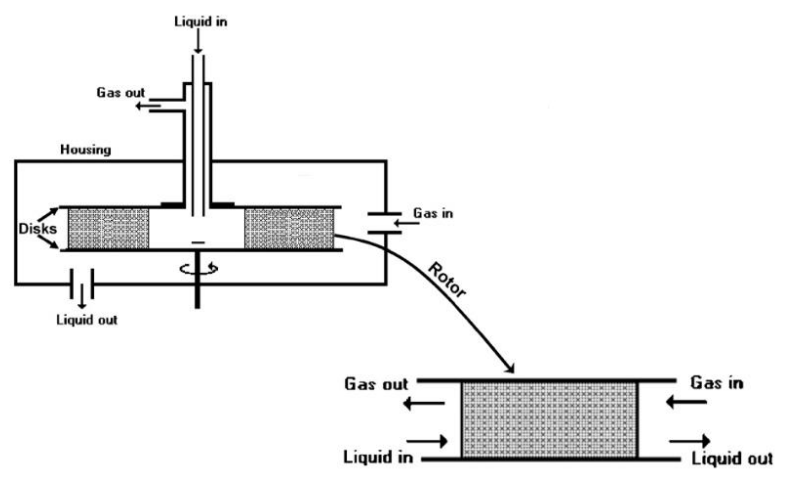

Figure 1 Sectional view of an RPB (Llerena-Chavez and Larachi 2009)

\subsection{Aim of this study}

As noted earlier, the predictive accuracies of mass transfer correlations for RPBs (Tung and Mah, 1985; Munjal et al., 1989a; Chen et al., 2006a; Chen et al., 2006b; Chen et al., 2006; Chen, 2011; Rajan et al., 2011; Luo et al., 2012), including modified mass transfer correlations for packed columns (Onda et al., 1968; Billets and Schultes, 1999) ought to be independently assessed. Joel et al. (2014) and Kang et al. (2014) attempted comparing and validating some of the correlations through process simulation. In their work, the mass transfer correlations were organised in sets - each set including correlations for predicting effective interfacial area, liquid and gas film mass transfer coefficients - and used separately in their model. Their RPB models were then validated using experimental data from RPB rigs. In their approach, several correlations are changed at a time in the model, and as such the individual performance of the correlations cannot be seen. What the authors (Joel et al., 2014; Kang et al., 2014) showed instead was that some sets of correlations were better than others. In this study, the aim is to provide a comprehensive review of existing correlations, compare and validate the correlations individually using experimental data obtained from literature. 
This study provides an extensive review and comparison of all published correlations for estimating different mass transfer parameters for RPBs, namely effective interfacial area, liquid and gas film mass transfer coefficients. As noted in Section 1.3, related study had been reported by Joel et al. (2014) and Kang et al. (2014). However, neither study included comparisons for gas film mass transfer coefficient and they considered the correlations in sets (See Section 1.3) and validated overall predictions of their RPB model and not specific predictions of the mass transfer parameters. This study will therefore address the following gaps identified from existing studies:

a. No information on performance of correlations for predicting gas film mass transfer coefficients for RPBs

b. No specific performance comparison of different mass transfer correlations for RPBs.

c. No data for the gas film mass transfer coefficient for RPBs. An assessment comparing liquid and gas film resistances to mass transfer for $\mathrm{CO}_{2}$ absorption in different MEA concentrations in an RPB absorber (Table 1) show that the gas film resistance could be over $10 \%$ of the overall resistance and cannot be ignored. Obtaining data for the gas film mass transfer coefficient is therefore essential. The gas film mass transfer coefficient data were derived from overall volumetric mass transfer coefficient $\left(K_{G} a\right)$ experimental data from the literature.

Table 1: Liquid and gas film resistances for an RPB absorber with MEA solvent*

\begin{tabular}{lll}
\hline MEA (wt \%) & Liquid film resistance $\left(\mathbf{P a ~}^{\mathbf{2}} \mathbf{~ s / m o l}\right)$ & Gas film resistance $\left(\mathbf{P a ~}^{\mathbf{2}} \mathbf{~ s / m o l}\right)$ \\
\hline 55 & 240490.2 & 23265.93 \\
\hline 75 & 172447.4 & 25305.32
\end{tabular}

*The liquid and gas film resistances have been obtained using conditions from Jassim et al. (2007) and reaction data from Ying and Eimer (2013). The liquid and gas film mass transfer coefficients were respectively obtained using Tung and Mah (1985) and Chen (2011).

\section{Methodology - model development}

The mass transfer parameters for the RPB derived from experimental measurements are reported in literature. In this study, selected RPB absorber rigs from literature used for deriving different mass transfer parameters are represented using models derived from first principle. The details of the selected rigs are given in Section 3 (effective interfacial area), Section 4 (liquid film mass transfer coefficient) and Section 5 (gas film mass transfer coefficient). In the RPB absorber model, different mass transfer correlations (See Sections 3, 4 \& 5) are used to predict mass transfer parameters. The predicted values for different correlations are then compared to their counterpart derived from experimental measurements for the selected case in the literature. The RPB absorber model, developed using gPROMS ModelBuilder ${ }^{\circledR}$, are represented using Equations 1-9. The thermo-physical properties are obtained using a combination of the electrolyte Non-Random Two-Liquid (elecNRTL) model in Aspen Plus ${ }^{\circledR}$ and data obtained from the literature. The elecNRTL model is accessed from gPROMS ModelBuilder ${ }^{\circledR}$ platform through the CAPE-OPEN interface. The model has been validated for $\mathrm{CO}_{2}$ absorption in MEA cases and presented in Oko et al. (2018). The following assumptions have been made in developing the model:

- Steady state conditions.

- One-dimensional differential mass and energy balances for liquid and gas phases

- Heat losses are neglected

- Heat and mass transfer are described using the two-film theory

- Reactions (where applicable) are accounted for using an enhancement factor in the overall mass transfer coefficient

\section{Material balance}

Gas phase: $0=\frac{1}{2 \pi r Z} \frac{\partial\left(G^{m} y_{i}\right)}{\partial r}-a N_{i}$

Liquid phase: $0=-\frac{1}{2 \pi r Z} \frac{\partial\left(L^{m} x_{i}\right)}{\partial r}+a N_{i}$ 


$$
\text { Liquid phase: } 0=-\frac{1}{2 \pi r Z} \frac{\partial\left(L^{m} h_{L}\right)}{\partial r}+a\left(h_{g / l}\left(T_{l}-T_{g}\right)-\Delta H_{r} N_{C O_{2}}-\Delta H_{v a p} N_{H_{2} O}\right)
$$

The molar fluxes for molecular components are obtained as follows based on the two-film theory:

$N_{i}=K_{G, i}\left(P_{g, i}-P_{i}^{e q}\right)$

The overall mass transfer coefficient $\left(K_{G, i}\right)$ comprise of mass transfer resistances on both the gas and liquid film (Eqn. 6). $P_{g, i}$ and $P_{i}^{e q}$ are respectively gas phase component partial pressure and component equilibrium partial pressure in the liquid phase.

$$
K_{G, i}=\frac{1}{\left(\frac{R T_{g}}{k_{G, i}}\right)+\left(\frac{H}{k_{L, i} E}\right)}
$$

The enhancement factor $(E)$ is used to account for the reactions in reactive cases. The enhancement factor $(E)$ is obtained on the basis of a pseudo first-order reaction regime as given in Eqn 7.

$$
E=\frac{\sqrt{k_{a p p} D_{L, C O}}}{k_{L, C O}}
$$

Finally, the interfacial heat transfer coefficient $\left(h_{g / l}\right)$ is obtained based on the Chilton-Colburn analogy:

$h_{g / l}=k_{L} \rho_{L} C_{p}^{L}\left(\frac{\lambda_{L}}{\rho_{L} C_{p}^{L} D_{L}}\right)^{\frac{2}{3}}$

\section{Case 1: Effective interfacial area}

\subsection{Experimental data and correlations}

In the literature, effective interfacial area data for RPB have been derived from measurements of $\mathrm{CO}_{2}$ absorption in $\mathrm{NaOH}$ solutions (Munjal et al., 1989b; Chen et al., 1997; Chen et al., 1999; Rajan et al., 2011; Yang et al., 2011; Luo et al., 2012a; Guo et al., 2014: Chu et al., 2015; Liu et al., 2015; Tsai and Chen, 2015; Luo et al., 2017) based on the approach proposed by Sharma and Danckwerts (1970). The reported data are mainly packing effective interfacial area; a few studies (Yang et al., 2011; Guo et al., 2014; Luo et al., 2017) reported the effective interfacial area for the different mass transfer zones, namely the packing, cavity and the end zones. It was found that the packing effective interfacial area makeup more than half of the total effective interfacial area (Yang et al., 2011). Recent studies have also investigated the effective interfacial area for novel packing designs, namely blade packing RPB with static baffles (Tsai and Chen, 2015), nickel foam packing (Chu et al., 2015) and structured wire mesh packing (Luo et al., 2017). Changes in the packing design was shown to have significant impact on the effective interfacial area (Tsai and Chen, 2015). The data from Luo et al. (2012a) was selected for this work. The Luo et al. (2012a) experiments comprised of a $1 \mathrm{M} \mathrm{NaOH}$ solution as the liquid phase and a mixed $\mathrm{CO}_{2}$ and $\mathrm{N}_{2}$ gas with approximately $10 \mathrm{~mol} \%$ of $\mathrm{CO}_{2}$ as the gas phase. The data is preferred to the data from other sources for the following reasons:

- The RPB used for obtaining the measurements (Table 2 Specification of RPB from is equipped with wire mesh packing. Wire mesh packings are proven to be very suitable for RPBs due to their better mass transfer performance and rigidity (Chen et al. 2006). Munjal et al. (1989b) data was obtained from an RPB with glass bead packing. Chen et al. (1997) and Chen et al. (1999) data were obtained from an RPB with corrugated disk packings.

- The packing is a traditional RPB design, wherein the packings are loaded uniformly across the radial depth of the RPB without gaps in-between packing rings, so called unsplit packing configuration (Figure 2). The packing is held between two disks and rotated by a single motor. Rajan et al. (2011) and Liu et al. (2015) on the other hand are based on split packing configuration, a relatively new packing design for 
187 For each of the cases (involving different correlations for predicting the effective interfacial area), the Tung and

Five correlations for predicting effective interfacial area in RPB have been evaluated in this study (Table 3 Correlations for calculating effective interfacial area in RPB). These include popular correlations for packed columns, namely Onda et al. (1968), Billets and Schultes (1999) and Puranik and Vogelpohl (1974), which have been used commonly for RPB design and modelling (Jassim et al., 2007; Joel et al., 2014; Kang et al., 2014). Others include Rajan et al. (2011) and Luo et al. (2012a) which were developed specifically for RPBs. Luo et al. (2017) proposed a new correlation for structured wire mesh packings as Luo et al. (2012a), developed for unstructured wire mesh packing, was not good enough for structured wire mesh packings (Luo et al., 2017). The new correlation (Luo et al., 2017) was not included in this study as we are focused on unstructured wired mesh packings. In addition, Lin et al. (2000) proposed a correlation for predicting the packing wetting area in RPBs. The correlation (Lin et al., 2000) was found to be obviously inaccurate for predicting the effective interfacial area and as a result was excluded from our evaluations.

Table 2 Specification of RPB from Luo et al. (2012a)

\begin{tabular}{|c|c|c|c|c|c|c|}
\hline \multicolumn{4}{|c|}{ Dimensions (mm) } & \multicolumn{3}{|c|}{ Packing } \\
\hline $\boldsymbol{r}_{\boldsymbol{i}}$ & $r_{o}$ & $r_{s}$ & $Z$ & Type & $a_{t}$ & $\varepsilon$ \\
\hline 78 & 158 & 248 & 50 & Wire mesh & 400 & 0.90 \\
\hline
\end{tabular}

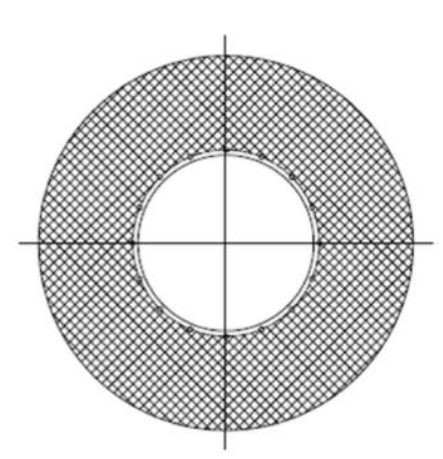

Figure 2 Unsplit packing configuration for RPB (Luo et al., 2012b)

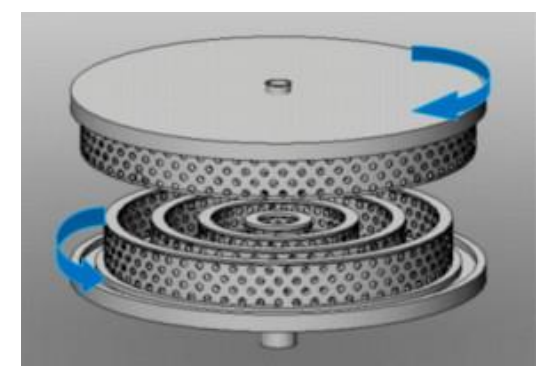

Figure 3 Split packing configuration for RPB (Liu et al., 2015) Mah (1985) and Chen (2011) were used for predicting the liquid and gas film mass transfer coefficients. The 
reported results are also mean values of the effective interfacial area across the flow direction in the RPB. The results in Figures 4 and 5 show that the predictions with Luo et al. (2012a) correlation provide the best agreement with experimental data. Modified Onda et al. (1968) correlation with "g" term replaced by " $r \omega^{2}$ " term which is widely used in literature for RPB design and modelling (Jassim et al., 2007; Joel et al., 2014; Kang et al., 2014) underpredicts the effective interfacial area by about 50\% and this increased with flowrate (Figure 5). The predictions of Onda et al. (1968) correlation with " $\mathrm{g}$ " term replaced by " $r \omega^{2}$ " term do not quite show impact of rotational speed on the effective interfacial area (Figure 4). More accurate prediction is obtained with modified Billets and Schultes (1999) correlation (i.e. with "g" term replaced by " $r \omega^{2}$ " term) although the deviation becomes increasingly large at high rotating speed. The predictions of Puranik and Vogelpohl (1974) correlation show nearly 50\% deviation. Comparing the predictions of Puranik and Vogelpohl (1974) with others at different rotating speed also highlight the impact of centrifugal acceleration. Although, Puranik and Vogelpohl (1974) correlation has been used successfully for packed columns, they clearly show poor prediction accuracy for RPBs. This partly because the correlation that do not have an acceleration term. Finally, the performance of Rajan et al. (2011) correlation which is developed for RPB was a bit surprising. The predictions deviated by nearly $50 \%$. The Rajan et al. (2011) correlation is based on split packing configuration and this is viewed as a major reason for the large error when the correlation is used for predicting the effective interfacial area for unsplit packing configuration in this study. It is recommended that the Rajan et al. (2011) correlation be used for split packing configuration cases only as it clearly underperforms for unsplit packing configuration case as shown in this study.

\section{Correlations}

\section{Comment}

Source

Onda et al. (1968)

These correlations have been modified for RPB by replacing the "g" term with " $\boldsymbol{r} \boldsymbol{\omega}^{2}$ " term.

Billets and Schultes

(1999)

$$
\frac{a}{a_{t}}=1.045\left(\frac{L_{m}^{*}}{a_{\mathrm{t}} \mu_{\mathrm{L}}}\right)^{0.041}\left(\frac{L_{m}^{2 *}}{\sigma_{L} \rho_{L} a_{t}}\right)^{0.133}\left(\frac{\sigma_{c}}{\sigma_{L}}\right)^{0.182}
$$

Puranik and

Vogelpohl (1974)
This do not have a "g" term. It was selected to know if good predictions are possible in RPB without explicitly accounting for acceleration.

$$
\begin{array}{ll}
\frac{a}{a_{t}}=54999\left(\frac{\rho_{L} d_{p} u_{L}}{\mu_{L}}\right)^{-2.2186}\left(\frac{u_{L}^{2}}{r \omega^{2} d_{p}}\right)^{-0.1748}\left(\frac{\rho_{L} d_{p} u_{L}^{2}}{\sigma_{L}}\right)^{1.3160} & \text { Rajan et al. (2011) } \\
\frac{a}{a_{t}}=66510\left(\frac{\rho_{L} d_{p} u_{L}}{\mu_{L}}\right)^{-1.41}\left(\frac{u_{L}^{2}}{r \omega^{2} d_{p}}\right)^{-0.12}\left(\frac{\rho_{L} d_{p} u_{L}^{2}}{\sigma_{L}}\right)^{1.21}\left(\frac{c^{2}}{(c+d)^{2}}\right)^{-0.74} \quad \text { Luo et al. (2012a) }
\end{array}
$$

These correlations are developed for RPB. Rajan et al. (2011) is based on split packing type RPB rotated by two separate motors. 


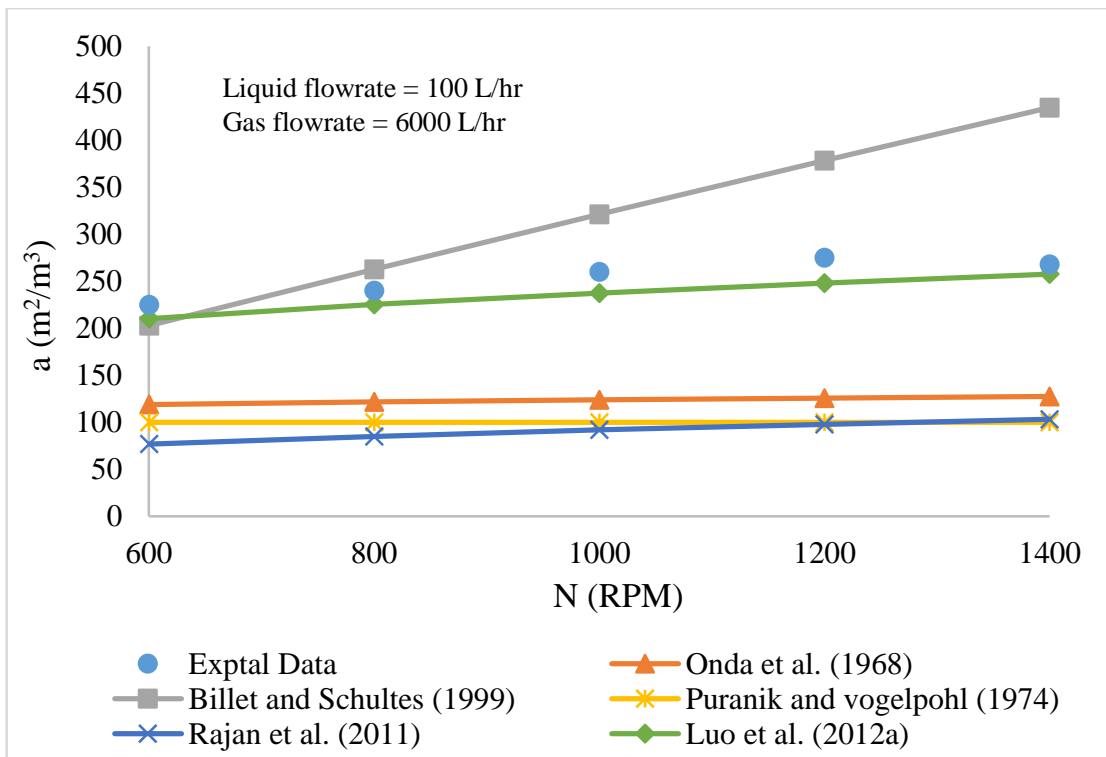

Figure 4 Predictions of different correlations for effective interfacial area at different RPM

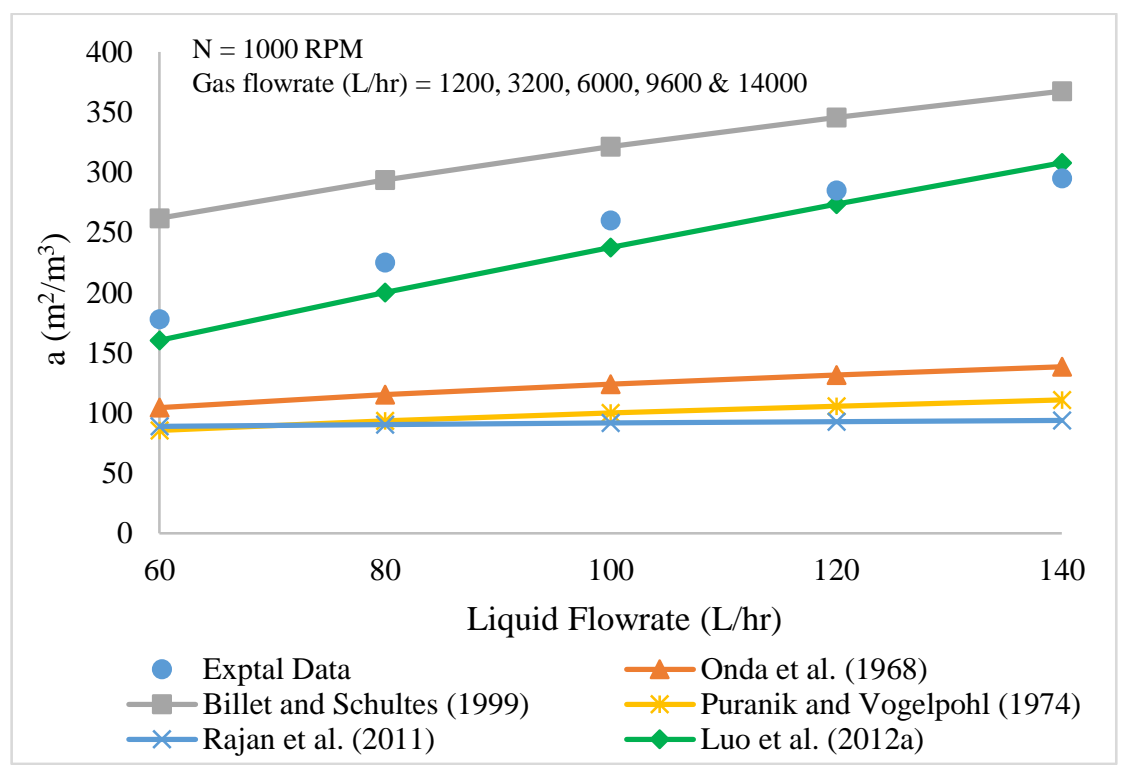

Figure 5 Predictions of different correlations for effective interfacial area at different liquid flowrate

4. Case 2: Liquid film mass transfer coefficient $\left(k_{L}\right)$

\subsection{Experimental data and correlation}

The study of liquid side mass transfer in RPBs is reported widely in literature, although it is the overall volumetric mass transfer coefficients $\left(K_{L} a\right)$ and the volumetric liquid side mass transfer coefficients $\left(k_{L} a\right)$ rather than the liquid film mass transfer coefficient (i.e. $k_{L}$ ) that are generally determined from experiments due to the difficulties in estimating the effective interfacial area in RPBs (Chen et al., 2005a; Chen et al., 2005b; Chen et al., 2006; Lin and Liu, 2007). The $K_{L} a$ is the overall driving force for the liquid phase while the $k_{L} a$ is the driving force for the liquid film only. The only existing $k_{L}$ data is reported by Luo et al. (2012b) and the experimental data has been selected for independently verifying different correlations for predicting liquid film mass transfer coefficients in this study. The data were derived from measurements of $\mathrm{CO}_{2}$ absorption in $\mathrm{NaOH}$ solutions based on the approach proposed by Sharma and Danckwerts (1970). The authors (Luo et al., 2012b) assumed a pseudo-first order reaction kinetics regime with mass transfer controlled by the liquid phase resistance. The liquid and gas phases were respectively $0.05 \mathrm{M} \mathrm{NaOH}$ solution and a mixed gas of $\mathrm{CO}_{2}$ and $\mathrm{N}_{2}$ with about $2 \mathrm{~mol} \%$ of $\mathrm{CO}_{2}$. A summary of the RPB parameters from Luo et al. (2012b) is given in Table 4. 


\begin{tabular}{|c|c|c|c|c|c|c|}
\hline \multicolumn{4}{|c|}{ Dimensions (mm) } & \multicolumn{3}{|c|}{ Packing } \\
\hline $\boldsymbol{r}_{i}$ & $r_{o}$ & $r_{s}$ & $Z$ & Type & $a_{t}$ & $\varepsilon$ \\
\hline 78 & 153 & 248 & 50 & Wire mesh & 500 & 0.96 \\
\hline
\end{tabular}

Presently, only five correlations (Table 5) for predicting the liquid film mass transfer coefficient have been published in literature (Tung and Mah, 1985; Munjal et al., 1989a; Chen et al., 2005a; Chen et al., 2005b; Chen et al., 2006); an elaborate model based on surface renewal theory has also been proposed by Ding et al. (2000). The reported correlations were developed from theoretical principle based on penetration theory (Tung and Mah, 1985; Munjal et al., 1989a) and the film theory (Chen et al., 2005a; Chen et al., 2005b; Chen et al., 2006). In this study, the performance of these five correlations, the summary of which are given in Table 5 Correlations for calculating liquid film mass transfer coefficient in RPB5, are evaluated. The modified Onda et al. (1968) was selected to demonstrate their performance for predicting $k_{L}$ for RPBs. The Tung and Mah (1985) correlation is simpler and requires fewer parameters compared to others. The Chen et al. (2006) correlation on the other hand is very elaborate, accounting for both the packing geometry and mass transfer in the end zones otherwise called the end effect phenomenon.

\section{Table 5 Correlations for calculating liquid film mass transfer coefficient in RPB}

$\begin{array}{lll}\text { Correlations } & \text { Source Comment }\end{array}$

\begin{tabular}{ll}
\hline$\frac{k_{L} d_{p}}{D_{L}}=0.918\left(\frac{\mu_{L}}{D_{L} \rho_{L}}\right)^{\frac{1}{2}}\left(\frac{L_{m}^{*}}{\mu_{L} a_{t}}\right)^{\frac{1}{3}}\left(\frac{a_{t}}{a}\right)^{\frac{1}{3}}\left(\frac{d_{p}^{3} \rho_{L}^{2} r \omega^{2}}{\mu_{L}^{2}}\right)^{\frac{1}{6}}$ & $\begin{array}{l}\text { Tung and } \\
\text { Mah (1985) }\end{array}$ \\
\hline
\end{tabular}

$$
\boldsymbol{k}_{L}=2.6 \frac{\pi L_{m}^{*}}{2 a \rho_{L} X}\left(\frac{\mu_{L}}{D_{L} \rho_{L}}\right)^{-\frac{1}{2}}\left(\frac{2 \pi L_{m}^{*}}{\mu_{L} a_{t}}\right)^{-\frac{2}{3}}\left(\frac{X^{3} \rho_{L}^{2} r \omega^{2}}{\mu_{L}^{2}}\right)^{\frac{1}{6}}
$$$$
\frac{k_{L} a d_{p}}{D_{L} a_{t}}=0.9\left(\frac{\mu_{L}}{D_{L} \rho_{L}}\right)^{0.5}\left(\frac{L_{m}^{*}}{\mu_{L} a_{t}}\right)^{0.24}\left(\frac{d_{p}^{3} \rho_{L}^{2} r \omega^{2}}{\mu_{L}^{2}}\right)^{0.29}\left(\frac{L_{m}^{2 *}}{\sigma_{L} \rho_{L} a_{t}}\right)^{0.29}
$$

Munjal et al. (1989a)

Onda et al. Modified for RPB by

(1968) replacing the "g" term with " $r \omega^{2}$ " term.

The correlations are developed for predicting $\boldsymbol{k}_{\boldsymbol{L}}$ in RPBs. They do not account for end effect and packing type

The Luo et al (2012a) correlations for effective interfacial area, demonstrated in Case 1 to give good predictions for unsplit and unstructured wire mesh packing, was used to predict the effective interfacial area for all the cases. Although, this potentially increases the prediction uncertainty, the results (Figure 6 and 7 ) show a reasonably good agreement for Tung and Mah (1985) and Chen et al. (2006). The gas film mass transfer coefficient was predicted 
for all cases using Chen (2011). The reported results are mean values of the liquid film mass transfer coefficient area across the flow direction in the RPB. The results further showed that the predictions of Tung and Mah (1985), Chen et al. (2005a), Chen et al. (2005b) and Chen et al. (2006) correlations for liquid phase mass transfer coefficient were in the order of $10^{-4}$. This is a typical range for liquid film mass transfer coefficients for RPBs which have been generally reported in the literature (Rao et al., 2004). The correlations of Onda et al. (1968) and Munjal et al. (1989a) respectively showed under-prediction and over-prediction in the orders of $10^{-5}$ and $10^{-3}$ at different rotating speed and liquid flowrate (Figures 8 and 9). The predictions of Onda et al. (1968) were in the typical range for the packed columns. It is concluded that modifying Onda et al. (1968) correlation by replacing the "g" term with " $r \omega^{2}$ " term do not result in good estimation of the liquid film mass transfer coefficient in RPBs.

Figure 6 Liquid film mass transfer coefficient at different RPM

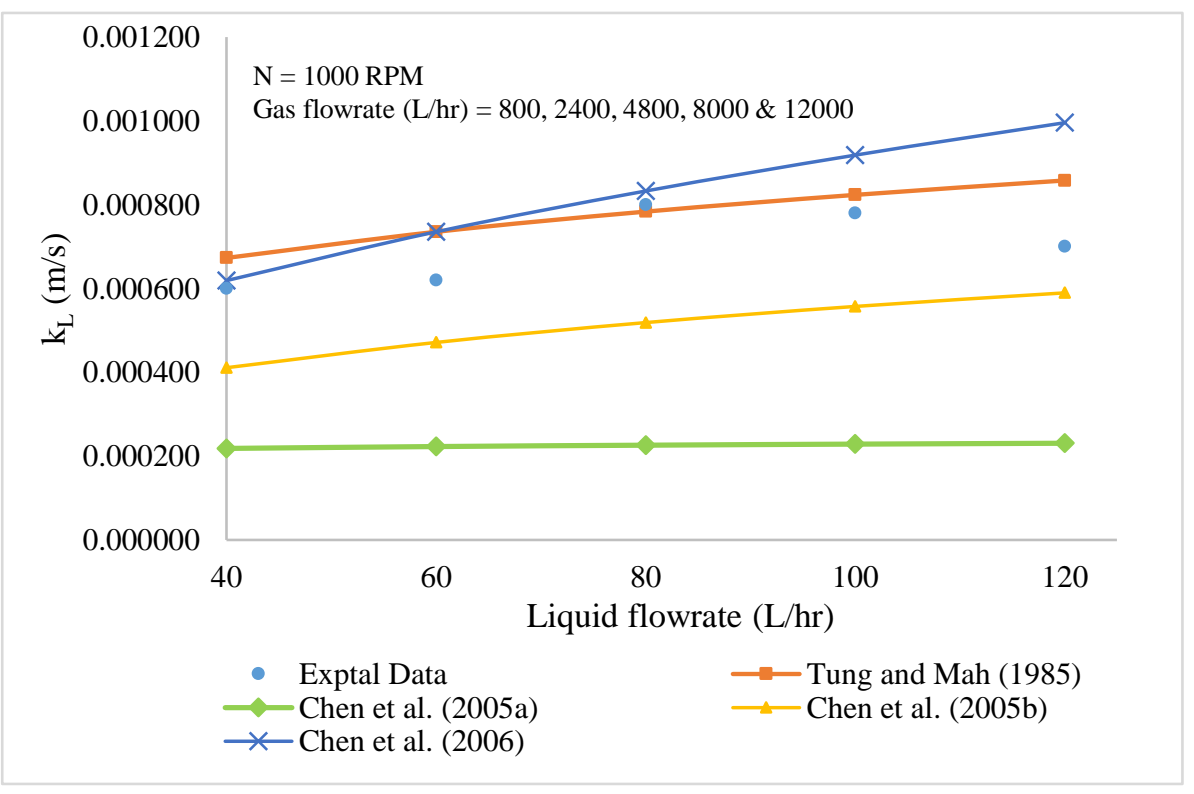

Figure 7 Liquid film mass transfer coefficient at different liquid flowrate

Comparing the Chen correlations (Chen et al., 2005a; Chen et al., 2005b; Chen et al., 2006), Chen et al. (2006) gave the best prediction compared to others. This is because apparently more extensive data set that cover different packing types, fluid types (Newtonian and non-Newtonian) and radial depth were used to develop the correlation. The Chen et al. (2006) and Tung and Mah (1985) correlations gave the best prediction for all conditions. The performance of Tung and Mah (1985) is particularly interesting as it is simpler, requiring fewer parameters and most of all does not account for the end effect and the packing type. The predictions of Chen et al. (2006) 
correlation, a supposedly more robust correlation that accounts for both end effect and packing type, tend to deviate as rotating speed and liquid flowrate increased. This deviation could be as a result of a combination of uncertainties from interfacial area and physical property predictions. Regardless, the maximum deviation was about $11 \%$ which is acceptable for most applications.

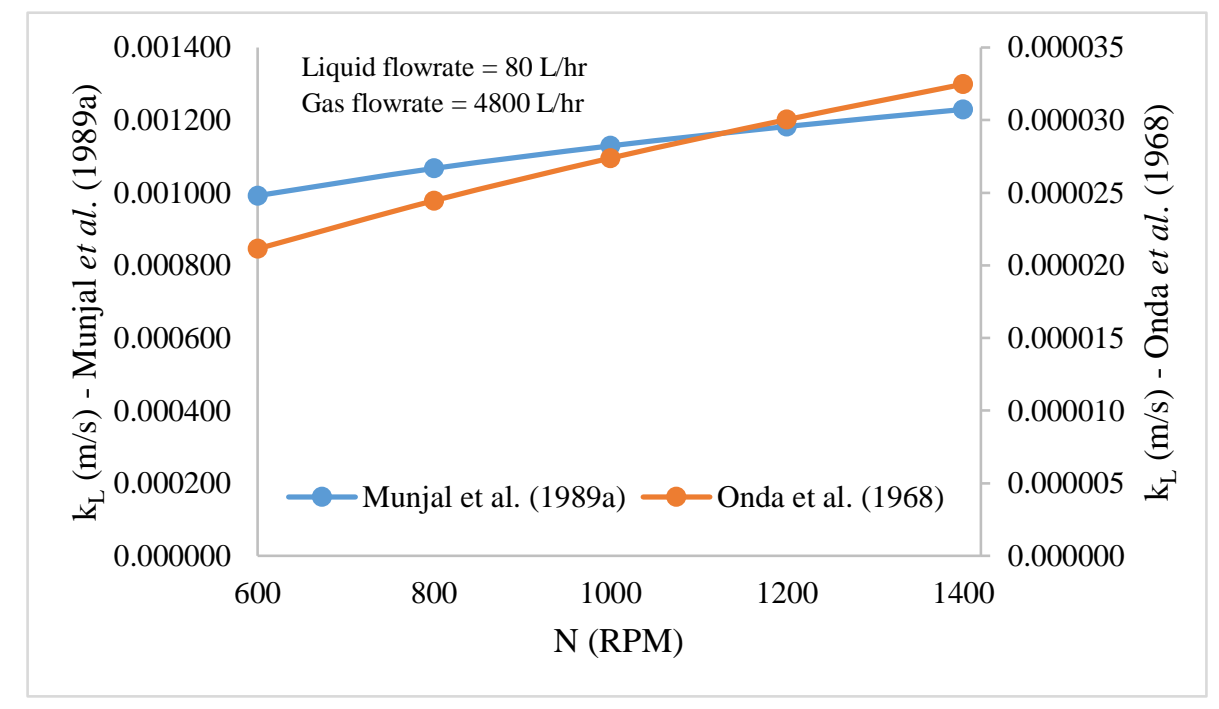

Figure 8 Liquid film mass transfer coefficient at different RPM

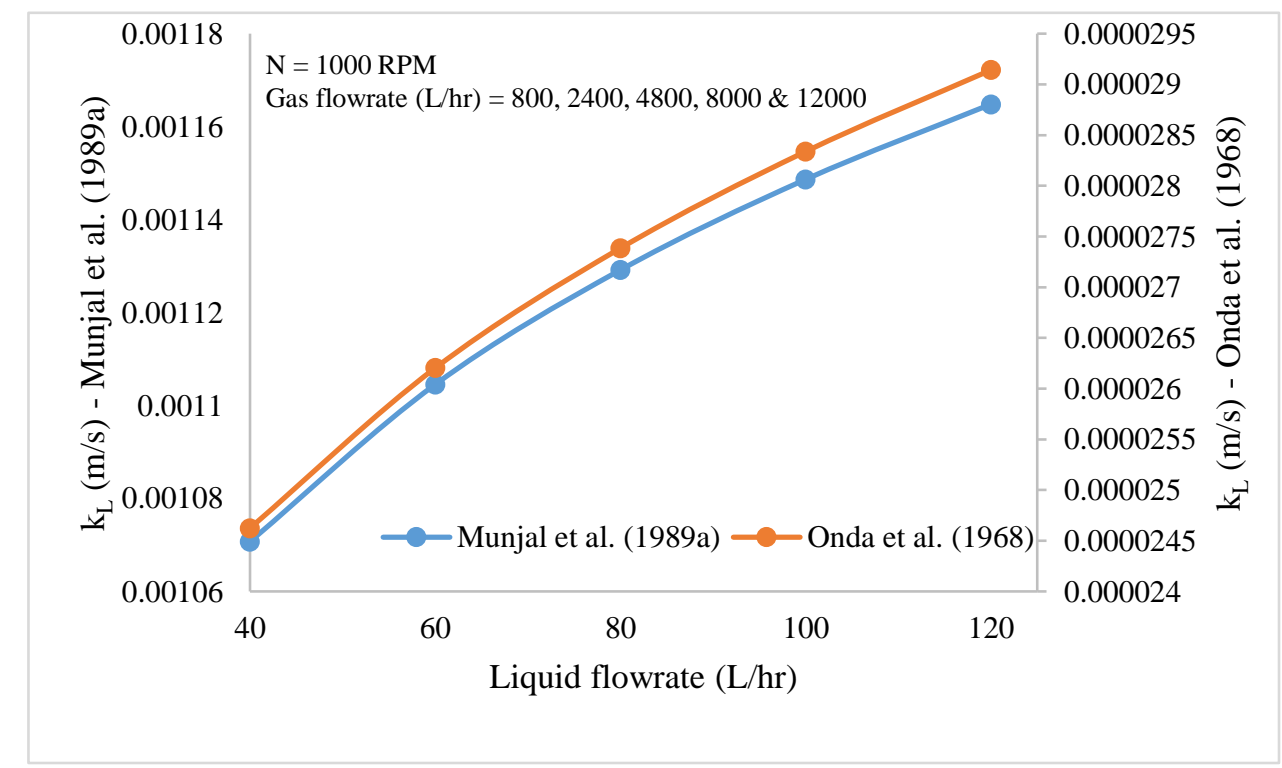

Figure 9 Liquid film mass transfer coefficient at different liquid flowrate

\section{Case 3: Gas film mass transfer coefficient $\left(k_{G}\right)$}

\subsection{Experimental data and correlations}

Experimental data for the gas film mass transfer coefficient $\left(k_{G}\right)$ calculation for RPBs are not available in the literature. What have generally been reported are overall volumetric mass transfer coefficient (i.e. $\left.K_{G} a\right)$. The $K_{G} a$ data are obtained using mass balance and the transfer unit concept (Liu et al., 1996; Chen and Liu, 2002; Lin et al., 2003; Lin et al., 2004; Chiang et al., 2009; Lin and Chu, 2015). The gas film volumetric mass transfer coefficient (i.e. $k_{G} a$ ) data have also been reported by Chen (2011). The $k_{G} a$ data from Chen (2011) was obtained based on the two-film theory (Eqn. 6) using a combination of published $K_{G} a$ data and $k_{L} a$ data predicted using the Chen et al. (2006) correlation. In this study, a similar approach as Chen (2011) has been adopted to obtain gas 
film mass transfer coefficient data $\left(k_{G}\right)$ from published $K_{G} a$ data alongside effective interfacial area and $k_{L}$ data predicted using Luo et al. (2012a) and Tung and Mah (1985) correlations respectively. Two independent sources, namely Lin et al. (2004) and Chiang et al. (2009), for $K_{G} a$ data were selected. The Lin et al. (2004) and Chiang et al. (2009) data involved isopropyl alcohol absorption in water and ethanol absorption in water respectively. Parameters of the RPB rigs used in both cases are summarized in Table 6.

Table 6 Specification of RPB from Lin et al. (2004) and Chiang et al. (2009)

\begin{tabular}{|c|c|c|c|c|c|c|c|}
\hline & \multicolumn{4}{|c|}{ Dimensions (mm) } & \multicolumn{3}{|c|}{ Packing } \\
\hline & $r_{i}$ & $r_{o}$ & $r_{s}$ & $Z$ & Type & $a_{t}$ & $\varepsilon$ \\
\hline Lin et al. (2004) & 35 & 80 & 150 & 35 & Wire mesh & 791 & 0.96 \\
\hline Chiang et al. (2009) & 20 & 40 & 60 & 20 & Wire mesh & 1024 & 0.944 \\
\hline
\end{tabular}

Existing correlations for the gas-side mass transfer coefficients are presented in Table 7. The correlations of Lin et al. (2004), Liu et al. (1996) and Chen and Liu (2002) are formulated for predicting overall volumetric mass transfer coefficient $\left(K_{G} a\right)$. The gas film mass transfer coefficient $\left(k_{G}\right)$ can be calculated from these correlations using Eqn 6. This will involve predicting several parameters namely the Henry's constant, enhancement factor (where applicable), liquid film mass transfer coefficient, effective interfacial area and physical properties such as density, viscosity and surface tension. The uncertainties in predicting these parameters could result in significant error in the gas film mass transfer coefficient. Therefore it was concluded that the correlations proposed in Lin $e t$ al. (2004), Liu et al. (1996) and Chen and Liu (2002) are not good options for predicting the gas film mass transfer coefficient and was therefore not considered for validation in this study.

Table 7 Correlations for calculating gas-side mass transfer coefficient in RPBs

\begin{tabular}{|c|c|c|}
\hline Correlations & Source & Comment \\
\hline$\frac{k_{G}}{a_{t} D_{G}}=K_{5}\left(\frac{V_{m}^{*}}{\mu_{G} a_{t}}\right)^{0.7}\left(\frac{\mu_{G}}{\rho_{G} D_{G}}\right)^{\frac{1}{3}}\left(a_{t} d_{p}\right)^{-2.0}$ & Onda et al. (1968) & $\begin{array}{l}\text { Correlation for predicting } \boldsymbol{k}_{\boldsymbol{G}} \\
\text { in packed columns }\end{array}$ \\
\hline$\frac{K_{G} a}{D_{G} a_{t}^{2}}=3.11 \times 10^{-3}\left(\frac{V_{m}^{*}}{\mu_{G} a_{t}}\right)^{1.163}\left(\frac{L_{m}^{*}}{\mu_{L} a_{t}}\right)^{0.631}\left(\frac{d_{p}^{3} \rho_{G}^{2} r \omega^{2}}{\mu_{G}^{2}}\right)^{0.25}$ & Liu et al. (1996) & \multirow{3}{*}{$\begin{array}{l}\text { These correlations are } \\
\text { developed for predicting } \\
\boldsymbol{K}_{\boldsymbol{G}} \boldsymbol{a} \text { in RPBs. The } \boldsymbol{k}_{\boldsymbol{G}} \text { can } \\
\text { then be derived from the } \\
\text { predicted } \boldsymbol{K}_{\boldsymbol{G}} \boldsymbol{a} \text { data using } \\
\text { Eqn } 6 .\end{array}$} \\
\hline$\frac{K_{G} a H^{0.27}}{D_{G} a_{t}^{2}}=0.077\left(\frac{V_{m}^{*}}{\mu_{G} a_{t}}\right)^{0.323}\left(\frac{L_{m}^{*}}{\mu_{L} a_{t}}\right)^{0.328}\left(\frac{d_{p}^{3} \rho_{G}^{2} r \omega^{2}}{\mu_{G}^{2}}\right)^{0.18}$ & Chen and Liu (2002) & \\
\hline$\frac{K_{G} a H^{0.315}}{D_{G} a_{t}^{2}}=0.061\left(\frac{V_{m}^{*}}{\mu_{G} a_{t}}\right)^{0.712}\left(\frac{L_{m}^{*}}{\mu_{L} a_{t}}\right)^{0.507}\left(\frac{d_{p}^{3} \rho_{G}^{2} r \omega^{2}}{\mu_{G}^{2}}\right)^{0.326}$ & Lin et al. (2004) & \\
\hline
\end{tabular}

$$
\frac{k_{G} a}{D_{G} a_{t}^{2}} V_{G}=K_{n}\left(\frac{V_{m}^{*}}{\mu_{G} a_{t}}\right)^{1.13}\left(\frac{L_{m}^{*}}{\mu_{L} a_{t}}\right)^{0.14}\left(\frac{d_{p}^{3} \rho_{G}^{2} r \omega^{2}}{\mu_{G}^{2}}\right)^{0.31}\left(\frac{L_{m}^{2 *}}{\sigma_{L} \rho_{L} a_{t}}\right)^{0.07}\left(\frac{a_{t}}{a_{p}^{i}}\right)^{1.4} \quad \text { Chen (2011) } \quad \begin{aligned}
& \text { The correlation is developed } \\
& \text { for predicting } \boldsymbol{k}_{G} a \text { in RPBs }
\end{aligned}
$$

Mukherjee et al. (2001) and Sandilya et al. (2001) had suggested that the gas phase undergoes a 'solid-body'-like rotation within the rotor of an RPB because of the drag offered by the packing and as a result suggested there was no enhancement of the gas film volumetric mass transfer resistance $\left(k_{G} a\right)$. Consequently, they concluded that the gas film volumetric mass transfer coefficient in RPBs was in similar range as that of packed columns. This was further demonstrated in Chen and Liu (2002) where it was shown that enhancements in $k_{G} a$ are mainly attributed to the interfacial area $(a)$, while $k_{G}$ remain in similar range as that of the packed columns. These conclusions have prompted the use of Onda et al. (1968) for predicting the gas film mass transfer coefficient in most published studies of RPBs (Joel et al., 2014; Kang et al., 2014). As a result, Onda et al. (1968) correlation for gas-film mass transfer coefficient was also evaluated in this study. 
343 The effective interfacial area has been predicted using the Luo et al. (2012a) and the liquid film mass transfer coefficient predicted using Tung and Mah (1985). The reported results are mean values of the gas film mass transfer coefficient across the flow direction in the RPB. The results of comparison between predicted values and experimental data (Figures 10 and 11) show that for the two experimental data considered in this study (Lin et al., 2004; Chiang et al., 2009), the Onda et al. (1968) correlation significantly over-predicts the $k_{G}$ in the RPBs for different rotating speed and gas flowrate. The predictions of Onda et al. (1968) are in the order of $10^{-1}$ in contrast to order of $10^{-2}$ values for the experimental data. Predictions of Chen (2011) on the other hand were in the order of $10^{-3}$. With the parameter $K_{n}$ updated from 0.023 to 0.23 , there was good agreement between the predictions of Chen (2011) and the experimental data for the two independent data sources in this study. Onda et al. (1968) correlation do not have a "g" term and as such they do not show the influence of centrifugal acceleration when they are used for predicting the $k_{G}$ in RPBs (Figure 10a and 11a). The experimental data as well as predictions of Chen (2011) both show that rotation enhances gas side resistance. Figures 10a and 11a both show that increasing rotating speed from 700-1600 RPM (Lin et al., 2004) and 600-1800 RPM (Chiang et al., 2009) respectively will enhance gas side resistance by up to $30 \%$. While $k_{G}$ appears to be in similar range as that of packed columns in agreement with the conclusions reached in Mukherjee et al. (2001) and Sandilya et al. (2001), their actual values are however affected by rotating speed. It is not possible to capture this impact when $k_{G}$ in RPBs are predicted using Onda et al. (1968).

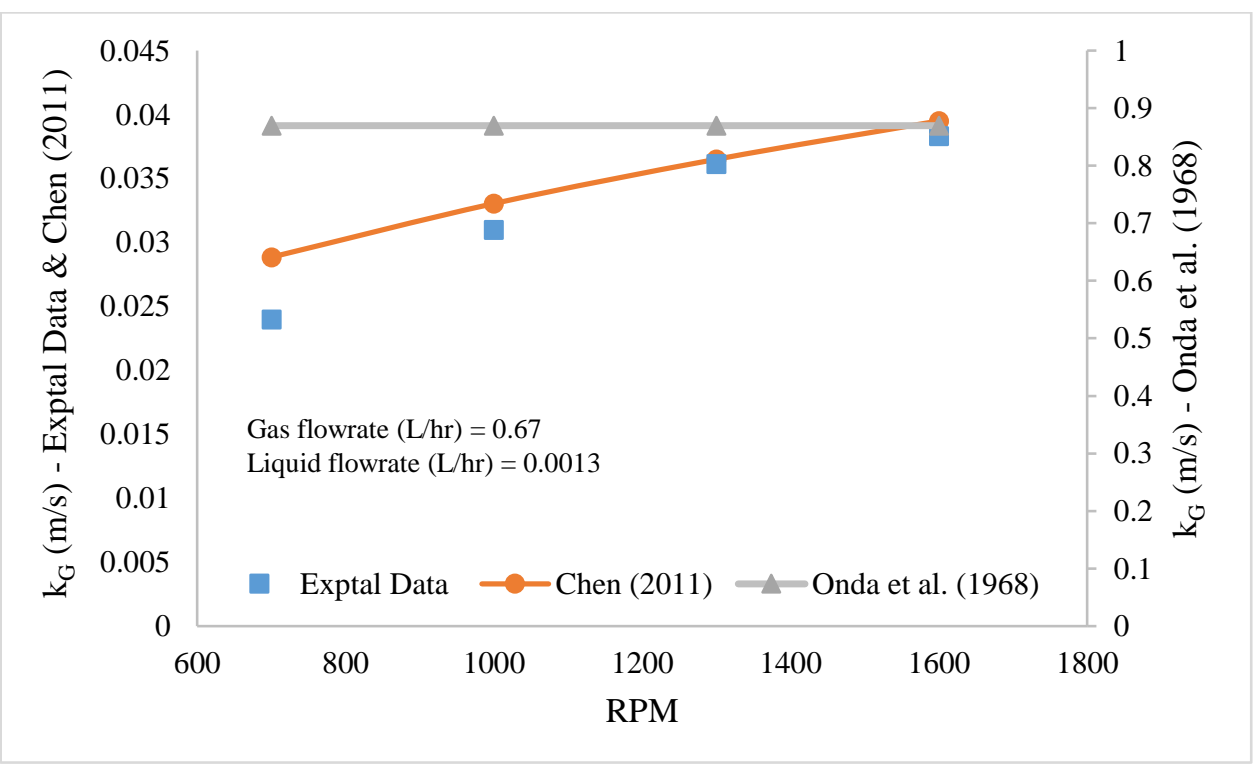




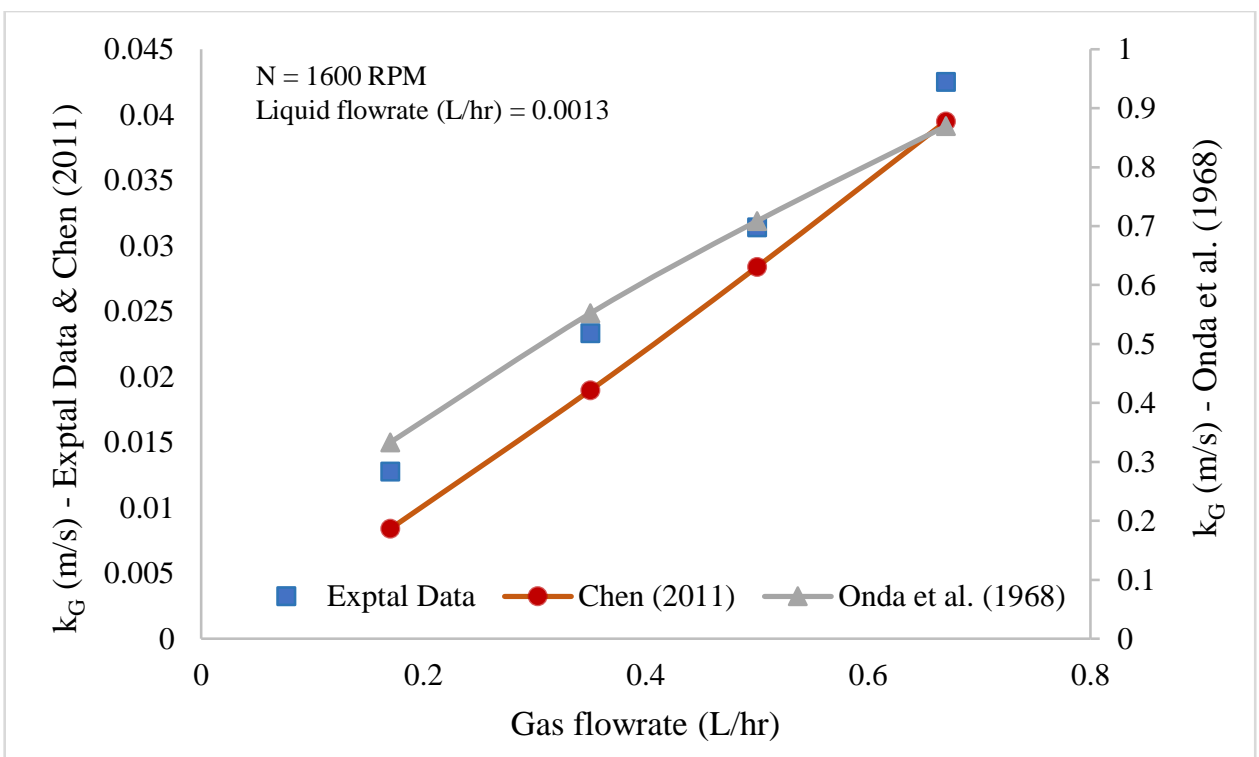

Figure 10b Comparisons to experimental data from Lin et al. (2004) data

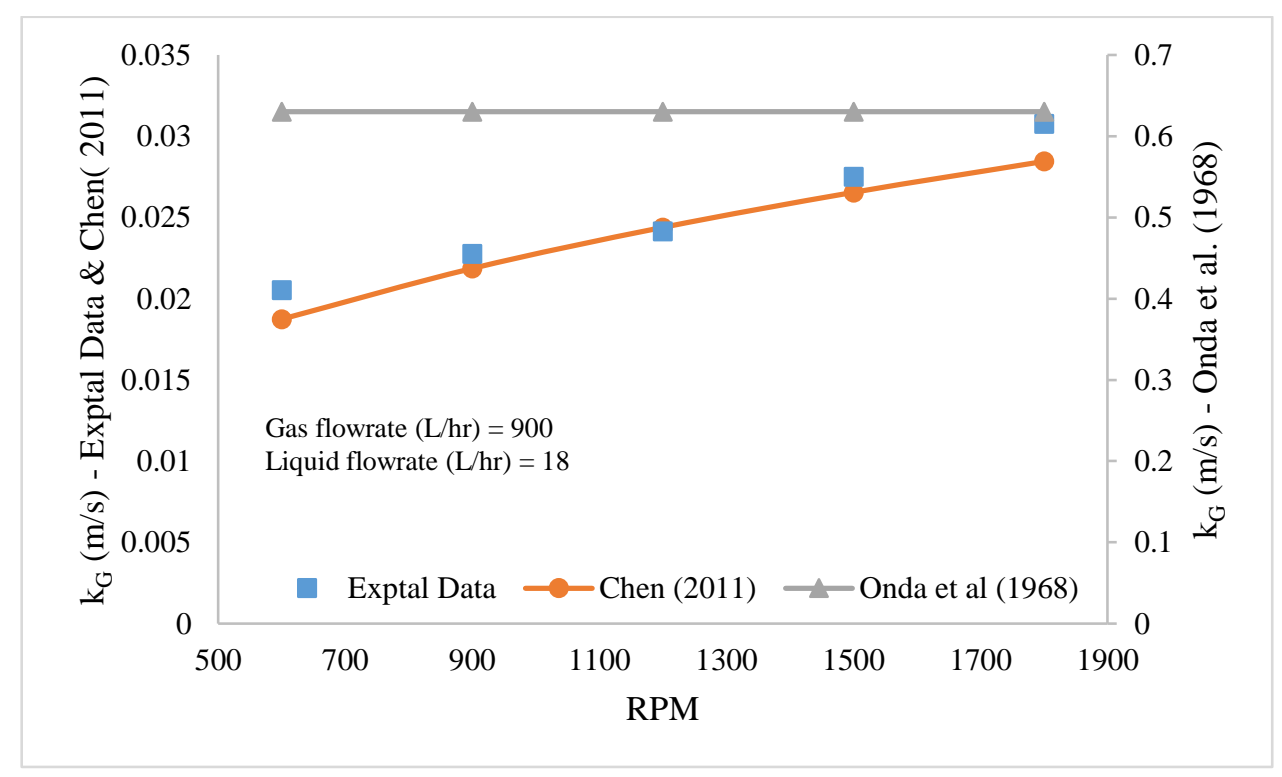




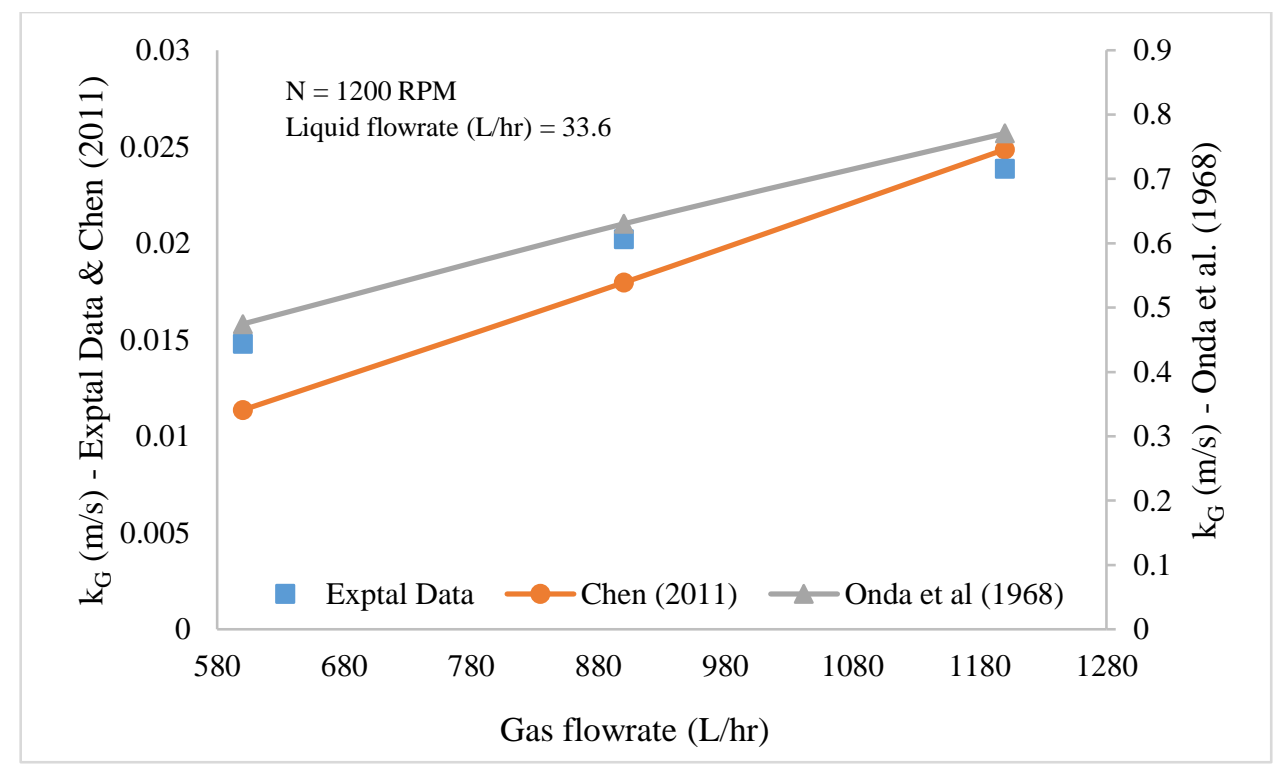

Figure 1la Comparisons to Chiang et al. (2009) data

\section{Conclusions and recommendations for future study}

The RPB is a promising technology that can greatly reduce the size and cost of packed columns used for absorption and desorption in solvent-based $\mathrm{CO}_{2}$ capture and natural gas treating processes. Mass transfer prediction in RPBs is not sufficiently proven because necessary correlations for doing this are generally few in literature and the prediction accuracy for existing correlations have not been demonstrated independently. In this study, an RPB model was developed in gPROMS ModelBuilder ${ }^{\circledR}$ and used to test and compare different correlations for the effective interfacial area, the liquid and gas film mass transfer coefficients at different rotating speed and liquid/gas flowrate. The results presented in this paper show that modified packed column mass transfer correlations with the " $g$ " term (i.e. gravitational acceleration) replaced with " $r w^{2}$ " (i.e. centrifugal acceleration) commonly used in literature for RPBs generally give poor predictions. The Tung and Mah (1985) correlation gave a good prediction of liquid film mass transfer coefficient in RPBs, slightly better than more complex correlations such as the Chen et al . (2006). The data of gas film mass transfer coefficient for RPBs were also derived from overall mass transfer coefficient $\left(K_{G} a\right)$ experimental data from the literature. This is the first report of gas film mass transfer data for RPBs in literature. Finally, we showed that Chen (2011) fitted the experimental data of gas film mass transfer coefficient better when the parameter $\left(K_{n}\right)$ is updated from 0.023 to 0.23 . The validity of the analysis and conclusions in this study are based on a single type of packing (unstructured wire mesh). With other packing types, namely expamet and retimet among others, the performance of the correlations may be very different. For instance, efforts in our group to use Luo et al. (2012a) for predicting effective interfacial area of expamet packings showed that the predicted values were out of range, although more data is needed to confirm this finding. The performance of the correlations should be demonstrated for other types of packings for RPB such as expamet and retimet as the relevant data become available.

\section{Acknowledgements}

The authors are grateful to the UK Engineering and Physical Sciences Research Council (EPSRC) (Ref: EP/M001458/2 and EP/N024672/1) for financing this research.

\section{References}

Agarwal, L., Pavani, V., Rao, D. and Kaistha, N. 2010. Process intensification in HiGee absorption and distillation: design procedure and applications. Ind Eng. Chem Res, 49, 46-58.

Chambers, H.H. and Wall, R.G. 1954. Some factors affecting the design of centrifugal gas absorbers. Trans Instn Chem Engrs 32, 96-107.

Chen, Y-S., Lin, F-Y., Lin, C-C., Tai, C. Y-D., and Liu, H.-S. 2006. Packing characteristics for mass transfer in a rotating packed bed. Ind. Eng. Chem. Res., 45, 6846-6853. 
Chen, Y.-S. 2011. Correlations of mass transfer coefficients in a rotating packed bed. Ind. Eng. Chem. Res., 50, $1778-1785$.

Chen, Y. S., Lin, C. C. and Liu, H.S. 2005a. Mass transfer in a rotating packed bed with viscous newtonian and non-newtonian fluids. Ind. Eng. Chem. Res., 44, 1043-1051.

Chen, Y. S. Lin, C. C. and Liu, H.S. 2005b. Mass transfer in a rotating packed bed with various radii of the bed. Ind. Eng. Chem. Res., 44, 7868- 7875.

Chen, H.H., Deng, X.H., Zhang, J.J. and Zhang, Y.J. 1999. The effective gas-liquid interfacial area and volumetric mass-transfer coefficient measured by chemical absorption method in rotating packed bed with multiple spraying under centrifugal force, Chem. React. Eng. Technol. 15, 97-103 (in Chinese).

Chen, H.H., Jian, Q.F. and Deng, X.H. 1997. The measurement of effective gas-liquid interfacial area of rotating bed by chemical absorption method, J. South China Univ. Technol. 27, 32-37 (in Chinese).

Chen, Y. S. and Liu, H.S. 2002. Absorption of VOCs in a rotating packed bed. Ind. Eng. Chem. Res., 41, 15831588 .

Chen, Y.-S, Hsu, Y.-C, Lin, C.-C, Tai, C.Y.-D and Liu H.-S. 2008. Volatile organic compounds absorption in a cross-flow rotating packed bed. Environ Sci Technol 42, 2631-2636.

Chiang, C-Y., Chen, Y-S., Liang, M-S., Lin, F.-Y. \& Tai, C. Y-D. and Liu, H.-S. 2009. Absorption of ethanol into water and glycerol/water solution in a rotating packed bed. Journal of the Taiwan Institute of Chemical Engineers, 40, 418-423.

Chu, G.-W., Sang, L., Du, X.-K., Luo, Y., Zou, H.-K., Chen, J.-F. 2015. Studies of $\mathrm{CO}_{2}$ absorption and effective interfacial area in a two-stage rotating packed bed with nickel foam packing. Chem. Eng. Process. 90, 3440.

Ding, X., Hu, X., Ding, Y., Wu, Y. and Li, D. 2000. A model for the mass transfer coefficient in rotating packed bed. Chem. Eng. Cam.., 178, 249-256.

Florin, N. and Fennell, P. n.d. Assessment of the validity of "approximate minimum land footprint for some types of $\mathrm{CO}_{2}$ capture plant" provided as a guide to the Environment Agency assessment of carbon capture readiness in DECC's CCR Guide for Application under Section 36 of the Electricity Act 1989.

Garcia, G.E.C., van der Schaaf, J. and Kiss, A.A. 2017. A review on process intensification in HiGee distillation. J Chem Technol Biotechnol., 92, 1136-1156.

Guo, K., Zhang, Z., Luo, H., Dang, J. and Qian, Z. 2014. An innovative approach of the effective mass transfer area in the rotating packed bed. Ind. Eng. Chem. Res., 53, 4052-4058.

Guo, F., Zheng, C., Guo, K., Feng, Y. and Gardner, N.C 1997. Hydrodynamics and mass transfer in cross-flow rotating packed bed. Chem Eng. Sci., 52, 3853-3859.

HiGee 2014. Available at: http://higeeusa.com/H2S.html [Accessed Dec., 2018].

IEAGHG, "Post-combustion $\mathrm{CO}_{2}$ capture scale-up study", 2013/05, February, 2013.

Jassim, M.S., Rochelle, G., Eimer, D., Ramshaw, C. 2007. Carbon dioxide absorption and desorption in aqueous monoethanolamine solutions in a rotating packed bed. Ind. Eng. Chem. Res., 46, 2823-2833.

Joel, A. S., Wang, M., Ramshaw, C., Oko, E. 2014. Process analysis of intensified absorber for post-combustion $\mathrm{CO}_{2}$ capture through modelling and simulation. Journal of Greenhouse Gas Control, 21, 91-100.

Kang, J.-L., Sun, K., Wong, D. S.-H., Jang, S.-S, and Tan, C.-S. 2014. Modelling studies on absorption of $\mathrm{CO}_{2}$ by monoethanolamine in rotating packed bed. International Journal of Greenhouse Gas Control, 25, 141150.

Kolawole, T., Attidekou, P., Hendry, J. and Lee, J. Comparative study of $\mathrm{CO}_{2}$ capture using counter and cross flow configurations in a rotating packed bed absorber using monoethanolamine (MEA). 14th International Conference on Greenhouse Gas Control Technologie (GHGT-14), October 21 -25, 2018, Melbourne, Australia.

Lawal, A., Wang, M., Stephenson, P. and Obi, O. 2012. Demonstrating full-scale post-combustion $\mathrm{CO}_{2}$ capture for coal-fired power plants through dynamic modelling and simulation. Fuel, 101, 115-128.

Lin, C.-C. and Liu, W.-T., 2007. Mass transfer characteristics of a high-voidage rotating packed bed. J. Ind. Eng. Chem., 13(1), 71-78.

Lin, C.-C., Chen, Y.-S. and Liu, H.-S. 2000. Prediction of liquid holdup in countercurrent-flow rotating packed bed. Trans IChemE, Vol. 78, Part A, 397-403.

Lin, C. C., Wei, T. Y., Liu, W. T. and Shen, K.P. 2004. Removal of VOCs from gaseous streams in a highvoidage rotating packed bed. J. Chem. Eng. Jpn., 37, 1471-1477

Lin, C.-C., Liu, W.-T. and Tan, C.-S., 2003. Removal of Carbon Dioxide by Absorption in a Rotating Packed 
Bed. Ind. Eng. Chem. Res., 42, 2381-2386.

Lin, C.-C. and Chu, C.-R., 2015. Mass transfer performance of rotating packed beds with blade packings in carbon dioxide absorption into sodium hydroxide solution. Separation and Purification Technology, 150, 196-203.

Liu, Y., Gu, D., Xu, C., Qi, G. and Jiao, W. 2015. Mass transfer characteristics in a rotating packed bed with split packing. Chinese Journal of Chemical Engineering, 23, 868-872

Liu, H.-S., Lin, C.-C., Wu, S.-C. and Hsu, H.-W., 1996. Characteristics of a rotating packed bed. Ind. Eng. Chem. Res., 35, 3590-3596.

Luo, Y., Chu, G.W., Zou, H.K. Zhao, Z.Q., Dudukovic, M.P. and Chen, J.F. 2012a. Gas-liquid effective interfacial area in a rotating packed bed. Ind. Eng. Chem. Res., 51, 16320-16325.

Luo, Y., Chu, G.-W., Zou, H.-K., Wang, F., Xiang, Y., Shao, L. and Chen, J.-F. 2012b. Mass transfer studies in a rotating packed bed with novel rotors: Chemisorption of $\mathrm{CO}_{2}$. Ind. Eng. Chem. Res., 51, 9164-9172.

Luo, Y., Luo, J.-Z., Chu, G.-W., Zhao, Z.-Q., Arowo, M. and Chen, J.-F. 2017. Investigation of effective interfacial area in a rotating packed bed with structured stainless steel wire mesh packing. Chemical Engineering Science 170, 347-354.

Mukherjee, R., Deb, D., Sandilya, P. and Rao, D.P., 2001. Gas-phase mass transfer in a centrifugal gas-liquid contactor with a stack of flexible disks as packing. In: Regel, L. L. and Wilcox, W. R. (eds) Processing by centrifugation. Boston, Massachusetts: Springer, 51-60.

Munjal, S., Dudukovic, M. P. and Ramachandran, P. 1989a. Mass-transfer in rotating packed beds-I: Development of gas-liquid and liquid-solid mass-transfer correlations. Chem. Eng. Sci., 44, 2245- 2256.

Munjal, S., Dudukovic, M. P. and Ramachandran, P., 1989b. Mass transfer in rotating packed beds-II. Experimental results and comparison with theory and gravity flow. Chemical Engineering Science, 44(10), 2257-2268.

Nascimento, J. V. S., Ravagnani, T. M. K. and Pereira, J. A. F. R. 2009. Experimental study of a rotating packed bed distillation column. Brazilian Journal of Chemical Engineering, 26(1), 219-226.

Oko, E. 2015. Study of power plant, carbon capture and transport network through dynamic modelling and simulation. PhD Thesis, School of Engineering, University of Hull.

Oko, E., Ramshaw, C. and Wang, M. 2018. Study of intercooling for rotating packed bed absorbers in intensified solvent-based $\mathrm{CO}_{2}$ capture process. Applied Energy 223 (2018), 302-316.

Rajan, S., Kumar, M., Ansari, M. J., Rao, D. P. and Kaistha, N., 2011. Limiting gas liquid flows and mass transfer in a novel rotating packed bed (HiGee). Ind. Eng. Chem. Res., 50, 986-997.

Rajan, S., Kumar, M., Ansari, M. J., Rao, D. P. and Kaistha, N., 2011. Limiting gas liquid flows and mass transfer in a novel rotating packed bed (HiGee). Ind. Eng. Chem. Res., 50, 986-997.

Ramshaw, C. and Mallinson, R.H. 1981. Mass Transfer Process. US Patent: 4283255

Rao, D. P., Bhowal, A. and Goswami, P.S. 2004. Process intensification in rotating packed beds (HIGEE): An appraisal. Ind. Eng. Chem. Res, 43, 1150-1162.

Sandilya, P., Rao, D. P. Sharma, A. and Biswas, G., 2001. Gas-phase mass transfer in a centrifugal contactor. Ind. Eng. Chem. Res., 40, 384-392.

Thiels, M., Wong, D. S. H., Yu, C.-H., Kang, J.-L., Jang, S. S., Tan, C.-S. 2016. Modelling and design of carbon dioxide absorption in rotating packed bed and packed column. In 11th IFAC Symposium on Dynamics and Control of Process Systems, including Biosystems. Trondheim, Norway.

Tsai, C.-Y. and Chen, Y.-S. 2015. Effective interfacial area and liquid-side mass transfer coefficients in a rotating bed equipped with baffles. Separation and Purification Technology 144, 139-145.

Tung, H. H.; Mah, R.S.H. 1985. Modeling liquid mass transfer in Higee separation process. Chem. Eng. Commun., 39, 147-153.

Yang, K., Chu, G., Zou, H., Sun, B., Shao, L. and Chen, J.-F., 2011. Determination of the effective interfacial area in rotating packed bed. Chemical Engineering Journal, 168, 1377-1382.

Ying, J. and Eimer, D. A. 2013. Determination and measurements of mass transfer kinetics of $\mathrm{CO}_{2}$ in concentrated aqueous monoethanolamine solutions by a stirred cell. Ind. Eng. Chem. Res. 52, 2548-2559. 